\title{
Mitigación de daños provocados por fauna silvestre en cultivos agrícolas en un bosque montano de Bolivia
}

Eddy Pérez L. ${ }^{1,2}$ \& Luis F. Pacheco ${ }^{1,3 *}$

1. Estación Biológica Tunquini, Instituto de Ecología, Casilla 10077, Correo Central, La Paz, Bolivia.

2. SMS - Medio Ambiente, Bloque Petrolero San Antonio, Petrobras Bolivia S.A., Av. Leigue Castedo No 1700, Santa Cruz, Bolivia; eddyug@gmail.com

3. Colección Boliviana de Fauna, Instituto de Ecología, Universidad Mayor de San Andrés, Casilla 10077, Correo Central, La Paz, Bolivia; luispacheco11@yahoo.com

* Correspondencia

Recibido 26-XII-2013. Corregido 15-VI-2014. Aceptado 23-VII-2014.

\begin{abstract}
Wildlife damage mitigation in agricultural crops in a Bolivian montane forest. Wildlife is often blamed for causing damage to human activities, including agricultural practices and the result may be a conflict between human interests and species conservation. A formal assessment of the magnitude of damage is necessary to adequately conduct management practices and an assessment of the efficiency of different management practices is necessary to enable managers to mitigate the conflict with rural people. This study was carried out to evaluate the effectiveness of agricultural management practices and controlled hunting in reducing damage to subsistence annual crops at the Cotapata National Park and Natural Area of Integrated Management. The design included seven fields with modified agricultural practices, four fields subjected to control hunting, and five fields held as controls. We registered cultivar type, density, frequency of visiting species to the field, crops lost to wildlife, species responsible for damage, and crop biomass. Most frequent species in the fields were Dasyprocta punctata and Dasypus novemcinctus. Hunted plots were visited 1.6 times more frequently than agriculturally managed plots. Crop lost to wildlife averaged $7.28 \%$ at agriculturally managed plots, $4.59 \%$ in plots subjected to hunting, and $27.61 \%$ in control plots. Species mainly responsible for damage were Pecari tajacu, D. punctata, and Sapajus apella. We concluded that both management strategies were effective to reduce damage by $>50 \%$ as compared to unmanaged crop plots. Rev. Biol. Trop. 62 (4): 1495-1507. Epub 2014 December 01.
\end{abstract}

Key words: crop damage, agricultural practices, local communities, conflict mitigation, Pecari, Dasyprocta, track stations.

El daño causado por fauna silvestre en cultivos agrícolas constituye una de las causas más frecuentes de conflicto dentro y fuera de las áreas protegidas (Newmark, Manyanza, Gamassa, \& Sariko, 1994; Fungo, 2011; Baldwin, Salmon, Schmidt, \& Timm, 2012). Una forma común de evaluar dichos conflictos es la caracterización de la percepción de la gente sobre las pérdidas causadas por la fauna silvestre (Newmark et al., 1994; Gillingham \& Lee, 2003; Weladji \& Tchamba, 2003), pero los productores tienden a sobre estimar sus pérdidas (Fungo, 2011); los estudios que cuantifican esas pérdidas de forma independiente son menos comunes. Trabajos realizados en África y Europa reportan pérdidas de 70 a $90 \%$ de las cosechas agrícolas, causadas principalmente por grandes mamíferos (Osborn \& Parker, 2002; Wilson, 2004; Sitati, Walpole, \& Leader-Williams, 2005). En Latinoamérica, existen pocos estudios cuantitativos sobre esta problemática, entre ellos podemos citar a Elías y Valencia (1984), Morales (2003), Pérez y Pacheco (2006), Romero-Balderas, Naranjo, Morales, y Nigh (2006), que reportan pérdidas entre 4 y $77 \%$ del cultivo. Las principales 
especies responsables de daños en cultivos agrícolas en zonas de bosque tropical son Dasyprocta sp., Cuniculus paca, Pecari tajacu, Tayassu pecari y Tapirus terrestris (NaugthonTreves, Mena, Treves, Álvarez, \& Redloff, 2003; Pérez \& Pacheco, 2006).

La mitigación de estas pérdidas se ha encarado desde diversos enfoques según el contexto social, económico y de conservación. El uso de técnicas sofisticadas, como cercas eléctricas y alarmas ha demostrado ser bastante eficiente, pero poco aplicable y sostenible en comunidades rurales, en comparación con técnicas tradicionales como la vigilancia y ahuyentamiento de individuos problema (O'ConnellRodwell, Rodwell, Rice, \& Hart, 2000; Osborn \& Parker, 2002).

En Bolivia, se han reportado pérdidas promedio de $18 \%$ en cultivos de maíz (Zea mays), en los valles interandinos del Área Natural de Manejo Integrado Apolobamba, ocasionadas principalmente por Tremarctos ornatus y Aratinga mitrata (Morales, 2003). Pérez y Pacheco (2006), registraron pérdidas entre 13 y $17 \%$ en cultivos de maíz, walusa (Colocasia esculenta, Xanthosoma sp.) y yuca (Manihot esculenta), con extremos de $100 \%$ de la producción en algunas parcelas en el Parque Nacional y Área Natural de Manejo Integrado (PN) Cotapata; e identificaron a $P$. tajacu y D. punctata como los principales responsables. Sin embargo, no conocemos trabajos que hayan implementado medidas con el objetivo de mitigar estas pérdidas. Este aspecto nos llevó a buscar alternativas de manejo para mitigar el conflicto, compatibilizando el contexto ambiental y socio-económico local (Treves, Wallace, Naughton-Treves, \& Morales, 2006). Es difícil erradicar el conflicto a gran escala, la meta principal debe ser disminuir las pérdidas de forma aceptable para los actores locales. Por lo anterior, nos propusimos evaluar experimentalmente dos técnicas de mitigación: prácticas agroculturales y cacería de control (ver Métodos). Cabe resaltar que la cacería de control fue prohibida al establecerse el PN Cotapata, por posibles efectos negativos sobre la biodiversidad. Sin embargo, no se ha reportado la extinción local de ninguna de las especies conflictivas en la zona, como consecuencia de esta práctica en el pasado (Roldán, 2001). La cacería implementada en este trabajo (autorizada por el Servicio Nacional de Áreas Protegidas, para el presente trabajo) se limitó a áreas de cultivo, sin incursiones al bosque.

En este contexto se implementaron las medidas de mitigación descritas y se evaluó su efectividad para reducir las pérdidas agrícolas provocadas por vertebrados silvestres. Los comunarios consideraron como reducción aceptable una disminución de $\sim 50 \%$, en relación a las cuantificadas entre 2001 y 2002, que alcanzaron 6,19 , y $23 \%$ para maíz, yuca y walusa, respectivamente, o una pérdida global de 16\% (Pérez \& Pacheco, 2006); es decir, la pérdida global promedio debía sea inferior al $8 \%$. De esta manera, este trabajo cubre los cuatros aspectos que integran el manejo de los daños por vida silvestre (VerCauteren, Dolbeer, \& Gese, 2012): definición del problema, ecología de las especies involucradas, aplicación de métodos de manejo y evaluación de los resultados. La discusión incluye las implicaciones para la conservación y posibles soluciones al conflicto.

\section{MATERIALES Y MÉTODOS}

Área de estudio: El estudio se llevó a cabo en la gestión agrícola 2003-2004, en el Parque Nacional y Área Natural de Manejo Integrado (PN) Cotapata $\left(68^{\circ} 18^{\prime}-16^{\circ} 20^{\prime} \mathrm{SW}, 68^{\circ} 03^{\prime}-16^{\circ}\right.$ $05^{\prime} \mathrm{NW}, 67^{\circ} 43^{\prime}-16^{\circ} 10^{\prime} \mathrm{NE}$ y $16^{\circ} 18^{\prime} \mathrm{SE}$ ), entre los municipios de Coroico y La Paz, Bolivia. El PN Cotapata cubre aproximadamente 58 000ha, entre 1200 y $5500 \mathrm{msnm}$. El trabajo se realizó en las comunidades de Chairo y Yucupi-Chimani, que albergaban $\sim 40$ familias en la cuenca del río Huarinilla, zona central del PN Cotapata. La topografía es accidentada, con pendientes usualmente $>45^{\circ}$, precipitación anual de $\sim 3000 \mathrm{~mm}$ y vegetación típica de bosques nublados y bosque yungueño (transición entre bosque nublado y bosque amazónico). La agricultura es la base de subsistencia de las dos comunidades y se desarrolla entre 1200 y $1600 \mathrm{msnm}$ (Ribera-Arismendi, 1995; 
Paniagua-Zambrana, Maldonado-Goyzueta, \& Chumacero-Moscoso, 2003). Los principales productos comercializados son cítricos y café; otros como maíz, walusa y yuca se destinan al autoconsumo, cultivándose en superficies $\sim 2000 \mathrm{~m}^{2}$ (Pérez \& Pacheco, 2006). Nuestro trabajo se centró en cultivos para autoconsumo y el daño por mamíferos, pues el daño por aves suele ser pequeño y difícil de cuantificar.

La agricultura de subsistencia generalmente se realiza en parcelas de $\sim 40 \mathrm{x} 40 \mathrm{~m}$, habilitadas mediante roza, tumba y quema, cultivadas por uno a tres años, hasta que decae su producción. Para este trabajo utilizamos parcelas recientemente habilitadas, de las cuales siete parcelas fueron destinadas a prácticas agroculturales, cuatro parcelas a cacería de control, y cinco parcelas fueron utilizadas como control. $\mathrm{Su}$ distribución fue concertada con los participantes, de forma que los tratamientos no interfirieran entre sí. Las características de cada tratamiento se describen a continuación:

Prácticas agroculturales (PAg): En siete parcelas se implementaron simultáneamente las siguientes prácticas: a) limpieza del estrato herbáceo y arbustivo en el borde de la parcela, con ancho aproximado de $10 \mathrm{~m}$; b) un cerco de olores con desechos humanos (fermentados de orín y ropa sucia) rodeando la parcela; c) un cerco de banderines de colores en todo el borde de la parcela, que la gente usa comúnmente como repelentes visuales. Los colores fueron cambiados mensualmente para evitar la habituación de los animales (O'Connell-Rodwell et al., 2000; Shivik, Treves, \& Callahan, 2003); y d) vigilancia regular (visitas día por medio) por el agricultor. El intervalo se estableció a partir de experiencias que mostraron más ataques en parcelas abandonadas por más de una semana (Pérez \& Pacheco, 2006).

Cacería de control (PCA): En estas parcelas los agricultores tenían permiso para cazar a los animales considerados dañinos. La frecuencia y método de cacería (escopeta calibre 16) fue definida por cada agricultor, siguiendo la costumbre antes del establecimiento del PN Cotapata.
Parcelas control (PCo): Aquellas sin tratamiento adicional a las prácticas habituales de cultivo realizadas por los agricultores de la zona.

Las parcelas con cacería de control estuvieron separadas de aquellas con prácticas agroculturales y las parcelas control por el río Huarinilla. Supusimos que el río dificultaría el tránsito de animales, reduciendo la interferencia entre tratamientos. Para la toma de datos se procedió de la siguiente manera:

Densidad de plantación: El área cultivada se estimó por el método de polígonos, con brújula y cinta métrica. La densidad de cultivo se estimó mediante conteo de plantas de las especies cultivadas en seis cuadrantes de $3 \times 3 \mathrm{~m}$, elegidos al azar.

Plantación atacada y especies responsables: Una vez por mes y con la ayuda del agricultor, se contó el número de plantas atacadas por tipo de cultivo y tratamiento, y se registró la especie responsable en base a las características del ataque. Se consideró como planta atacada aquella cuya producción sería nula como consecuencia del ataque.

Para evaluar la frecuencia de visitas de las especies, se instalaron estaciones de registro de huellas (huelleros) de $1 \mathrm{~m}^{2}$ con tierra tamizada para la impresión de huellas y con poca modificación en relación a la pendiente. Se instaló un huellero cada $10 \mathrm{~m}$ en el perímetro de cada parcela, por lo cual su número dependió del tamaño de la parcela. Se instalaron 10 huelleros similares en un transecto de $300 \mathrm{~m}$, desde el borde del cultivo al interior del bosque más próximo, para evaluar la presencia de las especies en los alrededores del cultivo. Los huelleros fueron activados y revisados al día siguiente, una vez por mes, desde el inicio al final del estudio.

Cosecha: La biomasa cosechada fue registrada por los propios comunarios en formularios pre-diseñados, debido a que la cosecha se realiza en varios eventos, de acuerdo a sus necesidades. 
Los propios comunarios registraron las especies cazadas en formularios pre-diseñados. Para cada animal cazado se anotó: especie, sexo, masa corporal, hora de captura y tiempo invertido por el cazador.

Para evitar el efecto del estadio de desarrollo del cultivo, la siembra se realizó entre agosto y octubre. Todos los cultivos estaban rodeados por bosque continuo, secundario o maduro.
La cuantificación de los daños se restringió a los cultivos más comunes: maíz, yuca y walusa; otras especies fueron excluidas por tamaño de muestra insuficiente. Los valores de densidad de plantación entre tratamientos se compararon mediante una prueba KruskallWallis (KW; Zar, 1999). Para estimar la frecuencia de visitas de animales al cultivo se utilizó la siguiente relación (Travaini, Pereira, Martinez-Peck, \& Zapata, 2003):

$$
\text { Frecuencia de visitas por especie }(\mathrm{FV})=\frac{\left(\mathrm{N}^{\mathrm{o}} \text { huelleros con huellas de la especie } \mathrm{Z}\right)}{\mathrm{N}^{\mathrm{o}} \text { total huelleros activados }} \times 100
$$

La FV se comparó entre tratamientos con una prueba de KW y prueba Bonferroni para el análisis a posteriori. También se comparó la $\mathrm{FV}$ a las parcelas de cultivo con las visitas a los bosques cercanos.
Para cada parcela se estimó el porcentaje de plantación perdida para cada tipo de cultivo mediante el cálculo:

$$
\text { Plantación perdida }=\frac{\left(\mathrm{N}^{\mathrm{o}} \text { total plantas atacadas }\right)}{\mathrm{N}^{\mathrm{o}} \text { total plantación estimada }} \times 100
$$

Inicialmente se comparó el porcentaje de plantación perdida entre parcelas con tratamiento y controles por tipo de cultivo, mediante la prueba KW; excluyendo las parcelas con $<300$ plantas de cada tipo de cultivo. Posteriormente, analizamos la relación entre plantación atacada y especie animal responsable; comparándose gráficamente el porcentaje de plantación atacada entre parcelas con tratamiento vs. parcelas control por mes (durante nueve meses). Calculamos la biomasa cosechada como la sumatoria de cosechas parciales (en $\mathrm{kg}$ ) para cada tipo de plantación y tratamiento, y la comparamos entre tratamientos con una prueba KW.

En todos los casos se graficaron medias y desviaciones estándar, a pesar de usarse análisis no paramétricos. La razón de esto es que las medias son más fáciles de visualizar que las medianas, como medidas de tendencia central en gráficos.

Como análisis exploratorio, utilizamos nuestros datos promedio de plantación atacada en cultivos protegidos con exclusiones y parcelas control (Pérez \& Pacheco, 2006) y los comparamos con los promedios del presente trabajo (cacería, prácticas agroculturales y controles). La comparación se realizó con una prueba de Mann-Whitney.

\section{RESULTADOS}

Densidad de plantación: El estudio comenzó y culminó con el mismo número de parcelas por tratamiento. La superficie cultivada varió entre 514 y $2380 \mathrm{~m}^{2}$, pero no difirió entre tratamientos $(\mathrm{KW} \mathrm{H}=0.75, \mathrm{p}>0.69$, g.1.=2). La densidad de plantación por tipo de cultivo sólo difirió entre tratamientos para el caso del maíz $(\mathrm{KW}, \mathrm{p}<0.01)$, que varió entre 0.66 y 9.42 plantas $/ \mathrm{m}^{2}$, con densidades menores en las parcelas sujetas a cacería; pero no difirió entre los tratamientos para la yuca, ni la walusa $(\mathrm{KW}, \mathrm{p}>0.5)$, cuyas densidades variaron entre 0.28 y 3.71 plantas $/ \mathrm{m}^{2}$ y 0.52 y 5.28 plantas $/ \mathrm{m}^{2}$, respectivamente.

Plantación atacada $\mathbf{y}$ especies responsables: Durante los 10 meses de seguimiento, se instalaron 11315 huelleros, 9017 alrededor 
de los cultivos y 2298 en bosque adyacente. El $6.3 \%$ de los huelleros instalados alrededor de cultivos fue visitado por animales, y casi el doble (12.1\%) de los huelleros activados en el bosque tuvo visitas. El $71.1 \%$ de las visitas a cultivos (568), correspondió a especies potencialmente dañinas: P. tajacu, D. punctata, C. paca, Dinomys branickii, Nasua nasua, Sapajus apella, ratones y aves (especies no identificadas); el $8.3 \%$ de las visitas correspondió a especies no dañinas (felinos, Dasypus novemcinctus, Didelphis sp., Eira barbara, Coendu prehensilis y Sciurus sp.) y el resto (20.6\%) no pudieron ser identificadas. Las visitas a los huelleros en bosque correspondieron en su mayoría a especies dañinas, pero en menor proporción que alrededor de los cultivos (53.1\%), con un $17.6 \%$ de visitas de especies no dañinas y un $29.4 \%$ de visitas no identificadas. A lo largo de todo el estudio, un promedio de $5.96 \%$ (DE=1.55) de los huelleros activados alrededor de las parcelas fue visitado cada mes, mientras que un promedio de $12.1 \%(\mathrm{DE}=1.96)$ de los huelleros activados mensualmente en bosque tuvo visitas. La elevada cantidad de huellas sin identificar se debe a que los animales muchas veces resbalan en el terreno y no dejan huellas claras.

Las especies con mayor frecuencia de visitas (FV) a los huelleros del bosque fueron D. punctata, P. tajacu y C. paca; alrededor de los cultivos fueron más frecuentes $D$. punctata y D. branickii (Fig. 1). La FV a los huelleros de las parcelas fue distinta entre los tratamientos, considerando todo el periodo de muestreo (KW $\mathrm{H}=11.6, \mathrm{p}=0.003$ ). Estas diferencias se dieron principalmente entre parcelas con prácticas agroculturales (PAg) y parcelas con cacería control (PCA; Bonferroni=0.002). Destacamos que la FV promedio en PCA $(\sim 13 \%)$ fue 1.6 veces mayor que en PAg $(\sim 8 \%)$.

Las especies con mayor FV a huelleros en bosques adyacentes a PAg fueron Dasyprocta sp. y P. tajacu, en bosques adyacentes a PCA fueron Dasyprocta sp. y D. branickii y las mayores FV en PCo correspondieron a Dasyprocta sp. y $P$. tajacu; aunque se tuvo una alta proporción de huellas no identificadas (Fig. 1). La frecuencia de visitas en bosques adyacentes a parcelas de cultivo no difirió entre los tres tratamientos $(\mathrm{KW} \mathrm{H}=3.12, \mathrm{p}=0.18)$.

Plantación atacada y especies responsables: Considerando las tres especies cultivadas bajo análisis, se perdió en promedio el 7.3\% de la plantación en PAg, $4.6 \%$ en PCA y $27.6 \%$ en PCo. La plantación atacada difirió para los cultivos de maíz y yuca, pero no para la walusa. En el caso del maíz se perdió menos en PAg que en PCA; y un porcentaje intermedio en las PCo. Para la yuca se perdió más en las PCo que en las que tuvieron algún tratamiento (PCA y PAg, Cuadro 1). Si bien el análisis estadístico no muestra diferencias significativas para el caso de la walusa, es destacable que dos parcelas control sufrieron pérdidas de $100 \%$. La potencia de la prueba debe haber sido afectada por la gran variabilidad en los daños. Es notorio que, para los cultivos de yuca, las PAg y PCA tuvieron pérdidas promedio de 8.4 y 15.1 veces menores que las ocurridas en PCo, respectivamente. En cambio, la proporción de plantación perdida (para los tres cultivos) en PAg y PCA fue 2.6 y 14.4 veces menor que en PCo, respectivamente. Sólo para el cultivo de maíz se tuvo resultado inverso a lo esperado, pues se perdió 2.1 veces más en PCA que en PCo. Es también destacable que el porcentaje promedio

CUADRO 1

Proporción (\%) de plantación atacada por tipo de cultivo y tratamiento. Se muestran las medianas y el rango intercuartílico (entre paréntesis)

\begin{tabular}{lcccc}
\multicolumn{1}{c}{ Cultivo } & Agrocultural (n) & Cacería (n) & Control (n) & KW H (P) \\
Maíz & $1.59(2.96)(7)$ & $8.62(9.51)(4)$ & $3.17(3.75)(5)$ & $7.66(0.022)$ \\
Yuca & $6.59(4.01)(6)$ & $0.00(7.59)(3)$ & $36.88(83.39)(3)$ & $6.47(0.039)$ \\
Walusa & $1.14(5.42)(7)$ & $3.23(2.36)(4)$ & $51.03(98.65)(4)$ & $1.27(0.53)$ \\
\hline
\end{tabular}



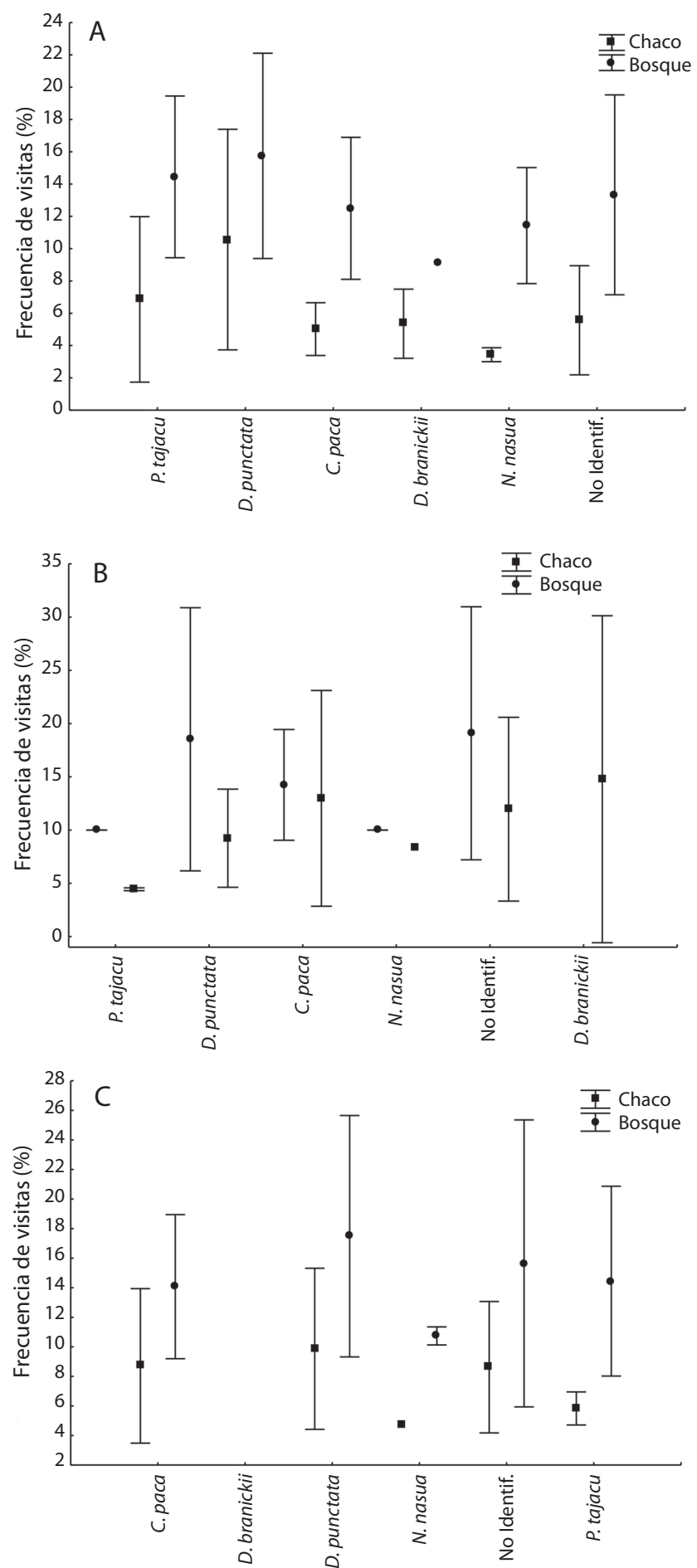

Fig. 1. Frecuencia de visitas (\% de huelleros con huella) de las distintas especies de animales a estaciones de registro de huellas en las parcelas de cultivo (chaco) y en el bosque adyacente: A) agrocultural, B) cacería y C) control. Se muestran la media \pm desviación estándar. 
de plantación perdida por causa de los animales silvestres en las parcelas bajo algún tratamiento fue de $5.95 \%$, mientras que el promedio para las PCo fue de $27.6 \%$. De esta manera, los resultados sugieren que los tratamientos aplicados cumplieron el objetivo de reducir las pérdidas absolutas en más del 50\%, aunque con gran variación entre parcelas. Sin embargo, ninguno de los tratamientos aplicados fue eficiente para reducir las pérdidas en cultivos de maíz.

El principal responsable de las pérdidas en cultivos de yuca y walusa, tanto en parcelas con tratamiento como en las parcelas control fue $P$. tajacu, seguido por D. punctata; éste último principalmente para plantaciones de yuca (Fig. 2A, Fig. 2B y Fig. 2C). Para el cultivo de maíz, la mayor proporción de plantación atacada en parcelas agroculturales fue causada por $D$. punctata, seguido de S. apella (Fig. 2A). No se pudo identificar a las especies responsables de la mayor parte del daño al maíz en parcelas con cacería de control, pero P. tajacu fue la especie que causó mayor daño en ese tipo de parcelas (Fig. 2B). Asimismo, P. tajacu y D. punctata fueron las especies cazadas con mayor frecuencia en PCA (tres individuos por cada especie, y un individuo de C. paca, durante los nueve meses de trabajo). Por último, en PCo la mayor proporción de plantas de maíz atacadas fue por P. tajacu (Fig. 2C).

Cosecha: El rendimiento de cosecha solamente difirió significativamente entre los dos tratamientos y las PCo solamente para la yuca, cuyo rendimiento de cosecha fue mayor en PCA (maíz: $\mathrm{KW} \mathrm{H}=5.14, \mathrm{p}=0.08$, yuca: $\mathrm{KW} \mathrm{H}=6.18, \mathrm{p}=0.045$, walusa: $\mathrm{KW} \mathrm{H}=2.32$, $\mathrm{p}=0.31$; Fig. 3). Sin embargo, es importante notar que la cosecha promedio de walusa en PCA fue más del doble que la promedio lograda en PCo o PAg (Fig. 3).

La comparación de plantación promedio atacada considerando los datos de Pérez y Pacheco (2006) y los del presente trabajo (Cuadro 2), muestran claramente que los tratamientos utilizados para controlar los ataques reducen efectivamente el daño, en comparación con parcelas control (sin tratamiento, MannWhitney $\mathrm{Z}=-2.55, \mathrm{p}<0.02$ ).

\section{DISCUSIÓN}

Las densidades de plantas para los tres cultivos durante este estudio fueron similares a las reportadas entre 2001-2003 en la misma zona (Pérez \& Pacheco, 2006), lo cual sugiere que existe uniformidad espacial y temporal en la densidad de plantación para cada cultivo en nuestra zona de estudio (maíz: $\sim 3$ a 4 plantas/ $\mathrm{m}^{2}$, yuca: $\sim 2$ plantas $/ \mathrm{m}^{2}$ y walusa: $\sim 2$ plantas/ $\mathrm{m}^{2}$ ). Esto sugiere que el presente estudio puede compararse también con el anterior.

En un análisis comparativo entre parcelas de cultivo y bosque adyacente es necesario destacar la frecuencia de visitas de P. tajacu, que causa la mayor proporción de pérdidas agrícolas en la zona (Pérez \& Pacheco, 2006; y

CUADRO 2

Comparación de los datos generales de pérdidas (promedio y DS) para los distintos tratamientos y controles de este estudio, y el de Pérez y Pacheco (2006) en la misma zona de trabajo. Se incluyen las principales especies que causaron los daños

\begin{tabular}{llllll} 
Cultivo & \multicolumn{1}{c}{ Agrocultural 2003-2004 } & \multicolumn{1}{c}{ Cacería 2003-2004 } & \multicolumn{1}{c}{ Exclusiones 2001} & \multicolumn{1}{c}{ Control 2003-2004 } & \multicolumn{1}{c}{ Control 2001} \\
Maíz & $1.43(1.36)$ & $8.44(5.50)$ & $4.27(3.06)$ & $4.00(4.48)$ P. tajacu & 5.94 (5.94) \\
& S. apella y D. punctata & P. tajacu, D. branickii, & N. nasua, aves y & y S. apella & D. punctata, aves, \\
& & N. nasua & S. apella & & N. nasua y ratones \\
Yuca & 4.57 (3.06) & $2.53(4.38)$ & $1.81(1.98)$ & $38.30(43.75)$ & 18.77 (33.15) \\
& P. tajacu y D. punctata & P. tajacu y D. punctata & D. punctata & P. tajacu y D. punctata & P. tajacu y D. punctata \\
Walusa & 15.83 $(37.19)$ & $2.81(1.89)$ & $0.35(0.59)$ & $40.53(54.28)$ & 23.32 $(43.52)$ \\
& P. tajacu & P. tajacu & P. tajacu & P. tajacu & P. tajacu \\
\hline
\end{tabular}

$\mathrm{NI}=$ no identificada. 

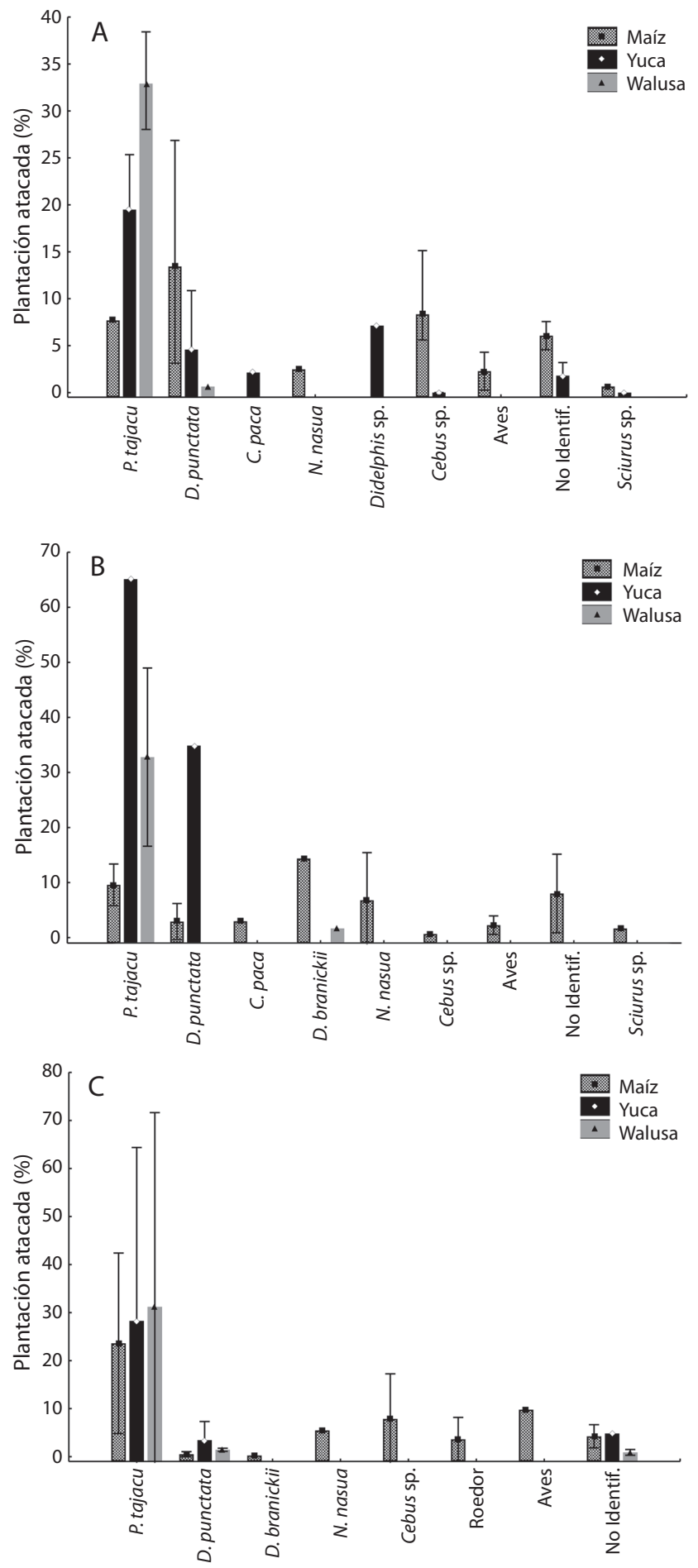

Fig. 2. Proporción de plantación atacada y especie responsable: A) agrocultural, B) cacería y C) control. 


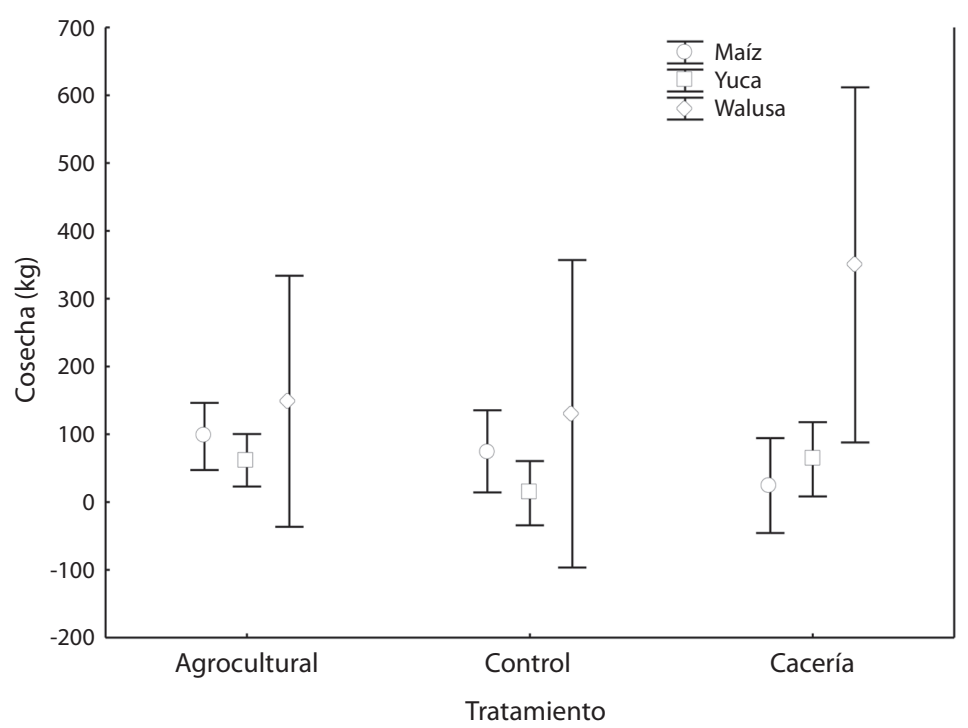

Fig. 3. Biomasa de cosecha por tratamiento y cultivo (se indica la media \pm desviación estándar).

este estudio). Esta especie fue más frecuente en bosques adyacentes que en parcelas de cultivo, aunque en pocas incursiones causa gran daño; algo similar ocurre para las otras especies, cuya presencia fue más frecuente en bosques que en parcelas, coincidiendo con lo reportado por Naughton-Treves et al. (2003), para la Amazonia peruana. Esta diferencia se mantiene incluso en las áreas de control, sugiriendo que las especies sólo se exponen a espacios abiertos para acceder a recursos alimenticios en alta densidad.

Las pérdidas promedio fueron mayores en las parcelas control $(\sim 27 \%)$, que en las parcelas con algún tratamiento ( $\sim 7 \%$ en las agroculturales y $4 \%$ en las parcelas con cacería de control), mostrando que los tratamientos cumplieron su objetivo, reduciendo las pérdidas cuatro a siete veces en relación a parcelas sin tratamiento. Pérez y Pacheco (2006) registraron pérdidas de $\sim 2 \%$ en parcelas cercadas con malla olímpica y de $16 \%$ en parcelas control. Es decir, aparentemente los ataques se redujeron durante este estudio, perdiéndose entre cuatro y siete veces menos plantación en parcelas con tratamiento que en parcelas control, en relación a lo reportado por Pérez y Pacheco (2006), cuando se perdió ocho veces menos plantación en parcelas con tratamiento en comparación a parcelas control. Esto sugiere que los métodos fueron eficientes en relación al objetivo planteado.

Si bien el uso de mallas de exclusión utilizado por Pérez y Pacheco (2006) parece ser más efectivo que los métodos evaluados en este trabajo, el costo de dicho método es más alto; lo cual lo hace poco sostenible en el tiempo (Osborn \& Parker, 2002; Shivik et al., 2003; VerCauteren et al., 2012). Adicionalmente, las exclusiones deben ser de tamaño suficiente, de forma que no afecten la producción de las plantas (Priston, 2009).

Tanto P. tajacu, como D. punctata atacan con mayor frecuencia cuando los productos están maduros, enero y febrero para maíz, mayo a agosto para walusa y yuca. Sin embargo, esto no implica que en el resto de los meses no existan daños que resulten menos perceptibles para los agricultores. Un patrón similar fue descrito por Naughton-Treves, Treves, Chapman y Wrangham (1998), para babuinos en Uganda, donde los ataques de los animales no resultaban distribuidos al azar en el tiempo, 
sino que existía una fuerte relación entre la proporción de ataques y la disponibilidad del recurso en las parcelas.

La falta de significancia estadística para las comparaciones de cosecha entre tratamientos y controles podría explicarse parcialmente por la alta variabilidad en la cosecha, realizada de forma artesanal con altas variaciones en calidad de semillas, tipos de suelos, prácticas agrícolas, entre otras, lo cual también fue reportado por Pérez y Pacheco (2006). También es posible que, la cosecha escalonada en el tiempo pueda afectar la biomasa total de cosecha; además que una proporción variable y no cuantificada de la producción nunca es cosechada, especialmente para walusa y yuca. Sin embargo, las diferencias absolutas en la biomasa cosechada entre parcelas con tratamientos y sin ellos son notorias, y la asociación entre el número de plantas perdidas y la biomasa de cosecha es consistente; todo esto sugiere fuertemente que los tratamientos son efectivos en reducir la pérdida de cosecha.

A pesar de la diferencia en tamaño y abundancia de las especies depredadoras, las pérdidas de yuca registradas en este estudio fueron superiores a las registradas en África por Naugthon-Treves et al. (1998) y por Hill (2000), pero las pérdidas en cultivos de maíz fueron inferiores a las reportadas por Weladji y Tchamba (2003) en África, aunque similares a lo reportado para el ANMI Apolobamba, Bolivia (Morales, 2003) y para México (RomeroBalderas et al., 2006). No se pudo realizar otras comparaciones para el caso de la walusa ya que no existen más estudios sobre daños por fauna silvestre sobre este cultivo que el de Pérez y Pacheco (2006). En todo caso, esto sugiere que el tipo de cultivo no es el que determina el daño, sino una compleja interacción de factores que incluyen el cultivo, las especies presentes en cada zona, sus abundancias, el momento en el tiempo y los esfuerzos que hace el agricultor para mejorar su cultivo y protegerlo de las especies dañinas. A su vez, esto implica que las medidas a adoptarse para mitigar el daño deben ser adaptadas localmente, de acuerdo al contexto local, además de las circunstancias que lleven a los animales a atacar un cultivo, en concordancia con lo propuesto por Baldwin et al. (2012) y VerCauteren et al. (2012).

La proporción de pérdidas de un determinado cultivo dependerá de la abundancia y tamaño de las especies depredadoras. Pocas o incluso una sola especie dañina pequeña, pero muy abundante, puede causar daños similares a los causados por una especie poco abundante, pero de gran porte. Hill (2000) y Gillingham y Lee (2003), reportan que los cultivos de maíz, yuca y arroz en África fueron el principal blanco de ataques, donde tanto monos, cerdos salvajes y aves fueron las principales responsables. En nuestro caso, el cultivo con mayor proporción de ataques para este estudio y el de Pérez y Pacheco (2006), fue la walusa, a pesar de ser atacado casi exclusivamente por P. tajacu. En cambio, el maíz fue atacado por seis (Pérez \& Pacheco, 2006) y ocho (este estudio) especies, pero resultó proporcionalmente menos atacado en parcelas control. Cabe resaltar que el maíz fue atacado en proporciones similares en todos los casos y tratamientos (incluso las exclusiones con malla), lo cual puede deberse a que algunas de las especies que lo atacan pueden trepar (Sapajus, Nasua, ratones) o volar (aves). También es interesante que las dos mejores formas de disminuir el ataque de $P$. tajacu a la walusa, fueron la cacería y las exclusiones.

Las implicaciones para la conservación, en relación a la cacería de control podría considerarse polémica (Dandy et al., 2012), especialmente tratándose de un área protegida; pero evidentemente fue bastante efectiva, exceptuando el caso de los cultivos de maíz. Además, la eficacia del método de control puede ser crucial a la hora de aplicarse a un problema en una región (Baldwin et al., 2012; VerCauteren et al., 2012). En este último caso podría deberse a que las especies depredadoras incluyen algunas que no son fáciles de detener, ni siquiera con cacería (monos, aves). Ojasti (2000) y Funes et al. (2006) mencionan que el control letal selectivo de individuos problema es una opción de manejo en conflictos fauna silvestre-poblaciones humanas, cuando 
los mismos tienen una repercusión fuerte sobre los intereses humanos. Nuestros resultados apoyan esta opinión, pues el control por cacería fue altamente selectivo a las especies (tres individuos de $D$. punctata y tres individuos de $P$. tajacu), que además pueden usarse como fuente de proteínas. En el pasado reciente (y posiblemente todavía ocurra) algunos agricultores utilizaban veneno, ante la prohibición de la cacería por parte de la administración del área protegida, con los potenciales efectos sobre especies carroñeras. Creemos firmemente que la cacería de control realizada exclusivamente dentro del área de cultivo es una buena opción de manejo del problema, pues aparte de sus beneficios en reducir depredación y proveer proteína animal al agricultor, sería altamente improbable que la cacería extinga localmente a las especies (Roldán, 2001).

Es importante señalar que la mitigación de las pérdidas no es sólo responsabilidad de las comunidades locales y la administración del área protegida, sino que existe también un grado de responsabilidad de actores indirectos, como organizaciones de conservación, organizaciones que operan con turismo en la zona, organizaciones políticas locales, entre otras, que de una $\mathrm{u}$ otra manera tienen a la conservación entre sus objetivos y/o reciben beneficios a partir de la conservación de estas áreas. Consecuentemente el éxito de la mitigación de estos conflictos también dependerá de factores socio-políticos, muchas veces ajenos al contexto local donde se encuentra el problema, como mencionan O'Connell-Rodwell et al. (2000). En este contexto, es esencial que todos los actores entiendan que estos conflictos no se pueden erradicar o solucionar definitivamente, por las siguientes razones:

- De conservación: Una forma de dar solución definitiva al problema sería la exterminación de las especies, lo cual es inaceptable en un área rural, sea dentro o fuera de áreas protegidas. Las estrategias de manejo deben dirigirse a compatibilizar las metas de conservación de la biodiversidad y el desarrollo sociocultural de los habitantes locales.

- Ecológicas: El control no letal de las especies conflictivas genera un desplazamiento del conflicto a otras zonas donde no se está realizando ningún tipo de control O'Connell-Rodwell et al. (2000). Al mismo tiempo, la efectividad de los métodos de control tiende a decrecer con el tiempo (Stevens \& Clark, 1998; O’Connell-Rodwell et al., 2000; Shivik et al., 2003). Así, las medidas de manejo deben considerar escalas espaciales y temporales amplias, y adecuar las medidas a los resultados de forma continua, sumado a un seguimiento poblacional de las especies conflictivas.

- Socio-cultural y económico: En general, los agricultores de la zona dedican poco tiempo al cuidado de sus cultivos por su bajo aporte en términos de mercado (Pérez \& Pacheco, 2006), lo cual reduce la factibilidad de disminuir el problema con base en mayor cuidado de los cultivos en el largo plazo. Sin embargo, es necesario mencionar que dichos cultivos forman parte importante de la dieta local.

Una de las formas más efectivas de control de las pérdidas parecería ser el trabajo comunitario. En nuestro sitio de estudio, esto podría hacerse con la rotación de gente en el cuidado de las parcelas o cultivando una parcela comunal de mayor tamaño. Observaciones preliminares en nuestro sitio de estudio sugieren que esta última opción sería efectiva, pues resulta en menor inversión de tiempo por parte de cada agricultor, en relación a parcelas unifamiliares. Esto coincide con lo reportado por Peters (1999), para el parque Ranomafana en Madagascar.

Con base en los resultados concluimos que:

- A través de las estrategias de manejo aplicadas (prácticas agroculturales y cacería de control), se logró reducir las pérdidas 
promedio de cultivos por animales silvestres en más del $50 \%$.

- Si bien las pérdidas son relativamente bajas en términos absolutos, el efecto para el agricultor llega a ser grande, registrándose inclusive pérdidas totales.

La mitigación del conflicto debe seguir los principios del manejo adaptativo. En este caso específico, deben manejarse varias estrategias complementarias e ir cambiando temporal y espacialmente, de forma que se logre una reducción del daño, se conserve a las especies, se evite el uso de venenos y se facilite el acceso a una fuente de proteínas para el agricultor. Todo esto debe encararse de forma que el agricultor acepte que el daño nunca será nulo. Todo el sistema de manejo debe estar bajo un estricto y robusto programa de monitoreo, que indique los resultados de las medidas aplicadas y su efecto sobre las poblaciones animales.

\section{AGRADECIMIENTOS}

A los comunarios de Chairo y Yucupi-Chimani, que participaron en el presente estudio. Este trabajo fue parte del financiamiento otorgado por John D. \& Catherine T. MacArthur Foundation, a la Estación Biológica TunquiniInstituto de Ecología UMSA, Bolivia. Jaime Jiménez y dos revisores anónimos comentaron una versión anterior de este manuscrito.

\section{RESUMEN}

El daño causado por fauna silvestre en cultivos agrícolas es una de las causas principales de conflicto dentro y fuera de áreas protegidas. Para un adecuado manejo del conflicto se requieren evaluaciones del daño y de la aplicación de distintos métodos de mitigación. El objetivo del trabajo fue evaluar la efectividad de prácticas agroculturales y cacería de control, para reducir daños por vertebrados silvestres en cultivos agrícolas anuales en el PN ANMI Cotapata. Se trabajó en siete parcelas con prácticas agroculturales, cuatro parcelas con cacería de control y cinco parcelas control, registrándose el tipo y densidad de cultivo, frecuencia de visitas de especies dañinas a huelleros, plantación perdida, especies responsables y biomasa de cosecha. Los resultados muestran que las especies más frecuentes fueron: Dasyprocta sp. y Dasypus novemcinctus, siendo estas 1.6 veces más frecuentes en parcelas con prácticas agroculturales. En promedio se perdió $7.3 \%$ de la plantación en parcelas agroculturales, $4.6 \%$ en parcelas sujetas a cacería de control, y $27.6 \%$ en parcelas control. Las principales especies responsables de las pérdidas fueron: P. tajacu, seguido por Dasyprocta sp. y S. apella. A través de estas estrategias de manejo, redujo las pérdidas en $>50 \%$, con lo cual se considera que los métodos puestos a prueba son útiles y se recomienda utilizarlos a gran escala con la consideración de que podría ser necesario innovar cada cierto tiempo, pues los animales silvestres tienden a acostumbrarse a los métodos de ahuyentamiento en ejecución.

Palabras clave: daño a cultivos, prácticas agroculturales, comunidades locales, mitigación de conflictos, Pecari, Dasyprocta, huelleros.

\section{REFERENCIAS}

Baldwin, R. A., Salmon, T. P., Schmidt, R. H., \& Timm, R. M. (2012). Wildlife pests of California agriculture: Regional variability and subsequent impacts on management. Crop Protection, 46, 29-37.

Dandy, N., Ballantyne, S., Moseley, D., Gill, R., Quine, C., \& Van Der Wal, R. (2012). Exploring beliefs behind support for and opposition to wildlife management methods: a qualitative study. European Journal of Wildlife Research, 58, 695-706.

Elías, D. J. \& Valencia, E. (1984). La agricultura latinoamericana y los vertebrados plaga. Interciencia, 9, 223-229.

Funes, M. C., Novaro, A. J., Monsalvo, O. B., Pailacura, O., Sanchez Aldao, G., Pessino, M., Dosio, R., Chehébar, C., Ramilo, E., Bellati, J., Puig, S., Videla, F., Oporto, N., González del Solar, R., Castillo, E., García, E., Loekemeyer, N., Bugnest, F., \& Mateáis, G. (2006). El manejo de zorros en la Argentina. Compatibilizando las interacciones entre la ganadería, la caza comercial y la conservación. In M. L. Bolkovic \& D. Ramadori (Eds.), Manejo de Fauna Silvestre en la Argentina: Programas de uso sustentable (pp. 151-166). Buenos Aires, Argentina: Dirección de Fauna Silvestre, Secretaría de Ambiente y Desarrollo Sustentable.

Fungo, B. (2011). A Review Crop Raiding Around Protected Areas: Nature, Control and Research Gaps. Environmental Research Journal, 5(2), 87-92.

Gillingham, S. \& Lee, P. (2003). People and protected areas: of local perceptions of wildlife crop-damage conflict in an area bordering the Selous Game Reserve Tanzania. Oryx, 37, 316-325. 
Hill, C. (2000). Conflict of interest between people and baboons: crop raiding in Uganda. International Journal of Primatology, 21, 299-315.

Morales, A. (2003). Daños causados por vertebrados silvestres en maizales de Pajan, K'apna y Wayrapata (ANMI Apolobamba, La Paz - Bolivia). (Tesis de Licenciatura). Universidad Mayor de San Andrés, La Paz, Bolivia.

Naughton-Treves, L., Treves, A., Chapman, C., \& Wrangham, R. (1998). Temporal patterns of cropraiding by primates: linking food availability in croplands and adjacent forest. Journal of Applied Ecology, 35, 596-606.

Naugthon-Treves, L., Mena, J. L., Treves, A., Álvarez, N., \& Redloff, V. C. (2003). Wildlife survival beyond park boundaries: the impact slash-and-burn agriculture and hunting on mammals in Tambopata Peru. Conservation Biology, 17, 1106-1117.

Newmark, W., Manyanza, D., Gamassa, D. M., \& Sariko, H. I. (1994). The conflict between wildlife and local people living adjacent to protected areas: Human density as a predictor. Conservation Biology, 8, 249-255.

O'Connell-Rodwell, C., Rodwell, T., Rice, M., \& Hart, L. A. (2000). Living with the modern conservation paradigm: can agricultural communities co-exist with elephants? A five-year case study in east Caprivi, Namibia. Biological Conservation, 93, 381-391.

Ojasti J. (2000). Manejo de Fauna Silvestre Neotropical. In F. Dallmeier (Ed.), SI/MAB, Series \# 5. Washington D.C.: Smithsonian Institution/MAB Biodiversity Program.

Osborn, F. \& Parker, G. E. (2002). Community-based methods to reduce crop loss to elephants: experiments in the communal lands of Zimbabwe. Pachyderm, $33,32-38$.

Paniagua-Zambrana, N., Maldonado-Goyzueta, C., \& Chumacero-Moscoso, C. (2003). Mapa de vegetación de los alrededores de la Estación Biológica Tunquini Bolivia. Ecología en Bolivia, 38, 15-26.

Pérez, E. \& Pacheco, L. F. (2006). Damage by large mammals to subsistence crops within a protected area in a mountain forest of Bolivia. Crop Protection, 25, 933-939.

Peters, J. (1999). Understanding conflicts between people and parks at Ranomafana Madagascar. Agricultural and Human Values, 16, 65-64.

Priston, N. E. C. (2009). Exclosure plots as a mechanism for quantifying damage to crops by primates. International Journal of Pest Management, 55(3), 243-249.
Ribera-Arismendi, M. O. (1995). Aspectos ecológicos del uso de la tierra y conservación en el Parque Nacional y Área Natural de manejo Integrado Cotapata. In C. Morales (Ed.), Caminos de Cotapata (pp. 1-84). La Paz: Instituto de Ecología.

Roldán, A. I. (2001). Manual de los mamíferos del PN ANMI Cotapata. Coroico, Bolivia: BIOTA, WCS, Audubon.

Romero-Balderas, K. G., Naranjo, E. J., Morales, H. H., \& Nigh, R. B. (2006). Daños ocasionados por vertebrados silvestres al cultivo de maíz en la selva Lacandona, Chiapas, México. Interciencia, 31, 276-283.

Shivik, J., Treves, A., \& Callahan, P. (2003). Nonlethal techniques for managing predation: primary and secondary repellents. Conservation Biology, 17, 1531-1537.

Sitati, N. W., Walpole, M. J., \& Leader-Williams, N. (2005). Factors affecting susceptibility of farms to crop raiding by African elephants: using a predictive model to mitigate conflict. Journal of Applied Ecology, 42, 1175-1182.

Stevens, G. \& Clark, L. (1998). Bird repellents: development of avian-specific tear gases for resolution of human-wildlife conflicts. International Biodeterioration \& Biodegradation, 42, 153-160.

Travaini, A., Pereira, J., Martinez-Peck, R., \& Zapata, S. (2003). Monitoreo de zorros colorados (Pseudalopex culpaesus) y grises (Pseudalopex griseus) en Patagonia: diseño y comparación de dos métodos alternativos. Mastozoología Neotropical, 10, 277-291.

Treves, A., Wallace, R. B., Naughton-Treves, L., \& Morales, A. (2006). Co-managing human-wildlife conflicts: A review. Human Dimensions of Wildlife, 11, $1-14$.

VerCauteren, K. C., Dolbeer, R. A., \& Gese, E. M. (2012). Identification and management of wildlife damage. In N. J. Silvy (Ed.), The wildlife techniques manual (7th Edition, Vol. 2, pp. 232-269). Baltimore: The John Hopkins University Press.

Weladji, R. \& Tchamba, M. (2003). Conflict between people and protected areas within the Benoue Wildlife Conservation area, North Cameroon. Oryx, 37, 72-79.

Wilson, C. J. (2004). Rooting damage to farmland in Dorset, southern England, caused by feral wild boar Sus scrofa. Mammal Review, 34, 331-335.

Zar, J. H. (1999). Biostatistical Analysis. New Jersey: Prentice-Hall International. 
